\title{
Feasibility of Shape-Memory Ni/Ti Alloy Wire Containing Tube Elevators for Transcrestal Detaching Maxillary Sinus Mucosa: Ex Vivo Study
}

\author{
Yanfeng Li Fuli Wang Pin Hu Jiadong Fan Yishi Han Bin Liu Tao Liu \\ Chunhao Yang Xiangmin Gu
}

Department of Stomatology, First Affliated Hospital of PLA General Hospital, Beijing, China

\author{
Key Words \\ Elevator $\cdot$ Osteotome sinus floor elevation $•$ Sinus mucosa - Shape-memory Ni/Ti alloy wire • \\ Transcrestal
}

\begin{abstract}
Background: Osteotome sinus floor elevation is a less invasive approach to augment an insufficient alveolar bone at the posterior maxilla for dental implantation. However, this approach has some limitations due to the lack of sinus lift tools available for clinical use and the small transcrestal access to the maxillary sinus floor. We recently invented shape-memory $\mathrm{Ni} / \mathrm{Ti}$ alloy wire containing tube elevators for transcrestal detaching maxillary sinus mucosa, and developed goat ex vivo models for direct visualizing the effectiveness of detaching sinus mucosa in real time during transcrestal maxillary sinus floor elevation. Methods: We evaluated our invented elevators, namely elevator 012 and elevator 014, for their effectiveness for transcrestal detaching maxillary sinus mucosa using the goat ex vivo models. We measured the length of sinus mucosa detached in mesial and distal directions or buccal and palatal directions, and the space volume created by detaching maxillary sinus mucosa in mesial, distal, buccal and palatal directions using the invented elevators. Results: Elevator 012 had a shape-memory $\mathrm{Ni} / \mathrm{Ti}$ alloy wire with a diameter of 0.012 inch, while elevator 014 had its shape-memory $\mathrm{Ni} / \mathrm{Ti}$ alloy wire with a diameter of 0.014 inch. Elevator 012 could detach the goat maxillary sinus mucosa in the mesial or distal direction for $12.1 \pm 4.3 \mathrm{~mm}$, while in the buccal or palatal direction for $12.5 \pm 6.7 \mathrm{~mm}$. The elevator 014 could detach the goat maxillary sinus mucosa for $23.0 \pm 4.9 \mathrm{~mm}$ in the mesial or distal direction, and for $19.0 \pm 8.1 \mathrm{~mm}$ in the buccal or palatal direction. An average space volume of $1.7936 \pm 0.2079 \mathrm{ml}$ was created after detaching the goat maxillay sinus mucosa in both mesial/distal direction and buccal/palatal direction using elevator 012; while the average space volume created using elevator 014 was $1.8764 \pm 0.2366 \mathrm{ml}$. Conclusion: Both two newly invented tube elevators could effectively detach the maxillary sinus mucosa on the goat ex vivo sinus models. Moreover, elevator 014 has advantages over the elevator 012 for the capability to detach sinus mucosa.
\end{abstract}

Y. Li, F. Wang and P. Hu are co-first authors. 


\section{Introduction}

Tooth loss at the posterior maxilla induces gradual bone resorption at the site of missing tooth $[1,2]$. The resulting insufficient alveolar bone at the posterior maxilla presents a big challenge for dental implantation. Maxillary sinus floor elevation techniques have been applied in the clinics for such cases for decades to augment the insufficient alveolar bone via elevating the maxillary sinus floor $[3,4]$. The success rate of dental implantation for such cases is still unsatisfactory [5], although significant progress has been made in the area of dental implantation by developing new techniques, implants, bone grafting materials and novel tools [6-15].

Maxillary sinus floor elevation could be performed via the lateral window approach or transcrestal approach [16]. The lateral window maxillary sinus floor elevation requires opening the front-outside wall of the maxillary sinus to provide an access to the maxillary sinus floor. Via this approach, a large scale of maxillary sinus mucosa could be detached, the tension produced by bone grafting could be reduced, and the height of sinus mucosa could be efficiently increased, thus enhancing the suitability for dental implantation. However, this invasive approach is not the first of choice in the clinics due to the trauma, long time required for surgery and healing, high cost, and technical complexity.

In 1994, Summers proposed to elevate the maxillary sinus floor via the transcrestal approach using osteotome, namely osteotome sinus floor elevation (OSFE) [3, 17]. For this technique and its derivatives, a hole was prepared at the osteotomy site in the alveolar, and this hole was gradually enlarged to prepare the implant socket till $1 \sim 2 \mathrm{~mm}$ beneath the maxillary sinus floor. Osteotome and mallet were then used to move the final $1 \sim 2 \mathrm{~mm}$ residual sinus floor bone and sinus mucosa into the cavity of maxillary sinus, thus elevating the maxillary sinus floor. The advantages for this approach include fewer traumas, simple and less invasive operation, and less postoperative discomfort. However, due to the visual limitations, OSFE has greater risks for maxillary sinus membrane perforations than the lateral window approach [18]. OSFE also has some limitations on the height achieved for elevated sinus floor. These limitations could be attributed to the small sized access hole prepared, which results in small scale of detached sinus mucosa and too much focused local stress to sinus mucosa simultaneously with the ridge augmentation. OSFE carries the risk for some complications, including tearing or perforation of the sinus membrane, infection, sinusitis, and unusually, benign paroxysmal positional vertigo $[19,20]$.

Various efforts have been made to overcome these limitations by improving the techniques and tools for transcrestal sinus floor elevation [6, 8, 12-14, 19-25]. Chen et al. [26] found that hydraulic pressure and pliable bone graft mixture could gently detach the soft tissue of sinus mucosa from bone in the sinus without the danger of perforation. This was attributed to the gentle pressure applied by hydraulic pressure and condensed grafting mixture instead of invading surgical instruments. The use of burs and condensers avoided surgical intrusion or perforation when a conically shaped sinus cavity access point was finished via a pinhole and tactile skill from the surgeon during the condensation stage. Only eight failures on implants were observed among 1557 implants during the 8-year study period. Mazor et al. [27] utilized a balloon to gently detach maxillary sinus mucosa via the uniform pressure generated by injecting water into the balloon. This significantly reduced the probability of maxillary sinus mucosa perforation, while increased the sinus lift height. Ahn et al. [28] used a series of specially designed reamers with one cutting and one reaming edge to prepare the osteotomy sites. They achieved a mean elevation height of $6.2 \pm 0.4 \mathrm{~mm}$ for all sinus floor elevation performed on 380 patients. Only $4.6 \%$ sinus membrane perforations were noticed, and the 2-year survival rate of placed implants was $95.4 \%$, while the success rate was $92.7 \%$ for those with thin sinus floors (i.e. $<4 \mathrm{~mm}$ ).

Nevertheless, it has been recognized that care should be taken when maxillary sinus floor needs to be elevated for more than $5 \mathrm{~mm}$, and currently transcrestal sinus floor elevation might not be suitable for those cases with severely insufficient heights of residual alveolar bones $[5,28,29]$. Moreover, the risk increases for perforation of sinus mucosa due to 
Li et al.: Ni/Ti Alloy Wire for Transcrestal Detaching Maxillary Sinus Mucosa

larger tension generated in sinus floor elevation. Studies on dead heads showed that the rate for perforation of sinus mucosa was $24 \%$ when OSFE was used to elevate the sinus floor for

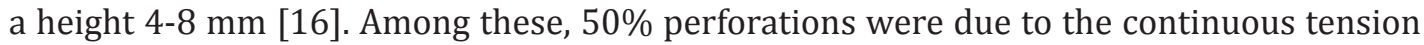
from the erupted mucosa. When perforation occurs, grafting materials could leak into the maxillary sinus if they are used to graft the elevated sinus floor. Thus, infection could happen in the maxillary sinus and even affect the healing around the implant, increasing the risk for the implant to fall off.

We designed and fabricated novel sinus lift elevators based on shape-memory alloy wire, namely shape-memory $\mathrm{Ni} / \mathrm{Ti}$ alloy wire containing tube elevators [19], especially for detaching maxillary sinus mucosa via the transcrestal approach. And we would like to hypothesize that our novel design could fully detach the maxillary sinus mucosa via the implant socket. They could be used to detach the maxillary sinus mucosa after reaching into the space through the implant socket prepared in the alveolar bone after osteotomy. Herein, we report the effectiveness of these two elevators to detach the maxillary sinus mucosa in goat ex vivo models.

\section{Materials and Methods}

The tools and instruments used for this study included bone saw (Tianjin Yutong Medical Instruments, China), osteotome instruments (Kelor, Germany), NewTom QR-DVT9000 computed tomography (CT), CV MM-II portable dental micromotor (Fushan, China), emery grinding needle (Sharp peak diamond abrasives company), shape-memory $\mathrm{Ni}$ /Ti alloy wire containing tube elevators including elevator 012 and elevator 014 (invented and fabricated by the authors, Fig. 1a-c) [19], MNT-M070 hammer (Shanghai, China), extraction forceps (Weirong, China), and ruler.

Forty three 1.5-2 years old goats (male or female, 20-30 kg) were purchased from the Animal Experimental Center at the First Affiliated Hospital of PLA General Hospital. They were healthy with fully developed maxilla and without maxillary sinus diseases. They were housed for one week before being sacrificed for the experiments. This study was approved by Institutional Animal Care and Use Committees.

The goats were sacrificed in the animal laboratory to obtain the entire heads. The heads were cleaned and then scanned using CT to examine and locate the maxillary sinus floors. The maxillofacial soft tissues were separated from the hard tissues after the CT scans. The mandibles were removed using bone saw. Four mark lines were drawn on each goat skull according to our previously developed protocol (data unpublished). Cuts were made along these marking lines using bone saw to obtain the primary goat ex vivo models (Fig. 2a). The maxillary pre-molar or molar root was extracted from the primary model, and implant socket was prepared in the maxilla (Fig. 2b) by simulating the clinical procedure of transcrestal maxillary sinus floor elevation until reaching the position of $1 \sim 2 \mathrm{~mm}$ beneath the maxillary sinus floor. The last 1 2 mm residual bone was removed using the osteotome technique to create a transcrestal access with a diameter of $4 \mathrm{~mm}$ to the maxillary sinus mucosa (Fig. 2c). The resulting models for real-time direct visualization of detaching sinus mucosa during sinus mucosa elevation were preserved in saline at $4^{\circ} \mathrm{C}$ and used within 24 hours for the following experiments.

The resulting 86 ex vivo goat sinus models (each goat provided two sinus models) were randomly divided into six groups: group A (16 models) for detaching the sinus mucosa in mesial and distal directions using elevator 012; group B (16 models) for detaching the sinus mucosa in buccal direction and palatal directions using elevator 012; group C (11 models) for detaching the sinus mucosa in both mesiodistal direction and buccal-palatal direction using elevator 012; group D (16 models) for detaching the sinus mucosa in mesial and distal directions using elevator 014; group E (16 models) for detaching the sinus mucosa in buccal direction and palatal direction using elevator 014; and group F (11 models) for detaching the sinus mucosa in both mesiodistal direction and buccal-palatal direction using elevator 014 .

To detach sinus mucosa in each group, the sinus mucosa was initially detached using the primary detaching knife (Fig. 1a) in the desired directions according to the group assignment, followed by detaching the sinus mucosa in the directions using the shape-memory wire in the desired elevator. The wire was used to detach the sinus mucosa via the movements of protruding, followed by rotation and then turn over (Fig. 3). During the detaching, the most front end of the wire was ensured to be closely against the sinus floor 


\section{Cellular Physiology Cell Physiol Biochem 2016;40:944-952 \begin{tabular}{l|l|l} 
and Biochemistry Published online: December 07, 2016 & $\begin{array}{l}\text { (c) 2016 The Author(s). Published by S. Karger AG, Basel } \\
\text { www.karger.com/cpb }\end{array}$
\end{tabular} \\ Li et al.: Ni/Ti Alloy Wire for Transcrestal Detaching Maxillary Sinus Mucosa}

bone. The operation was gently performed to avoid perforation to the sinus mucosa during the detaching. When the most front end of the wire reached the farthest position or the mucosa was perforated, the length of the detached sinus mucosa in that particular direction was recorded after the detaching operation was stopped. For groups $\mathrm{C}$ and $\mathrm{F}$, the mucosa $(\mathrm{n}=11)$ was mesiodistally, buccally and palatally detached using the elevator according to the group assignment. The operation of detaching sinus mucosa was stopped when the most front end of the wire reached the farthest position in every direction or the mucosa was perforated. Before and after detaching sinus mucosa for these two groups, saline was injected into the space under the sinus mucosa (Fig. 4 ) to obtain the space volume under the initial sinus mucosa $\left(\mathrm{V}_{1}\right)$ and the space volume under the elevated sinus mucosa $\left(\mathrm{V}_{2}\right)$. The space volume created by transcrestal sinus lift was calculated by subtracting $\mathrm{V}_{1}$ from $\mathrm{V}_{2}$. All the detaching operations were conducted firmly, evenly, and gently by the same laboratory personnel.

The data were reported as mean \pm standard deviation and analyzed using commercially available statistics software SPSS 17.0. The statistical difference level was set at $p=0.05$, while the statistically significant difference level was set at $p=0.01$.

\section{Results and Discussion}

We has designed goat ex vivo sinus models for direct visualization of transcrestal detaching maxillary sinus mucosa in real time (data unpublished). In this work, we used the same method to establish goat ex vivo models (Fig. 2) for evaluating the effectiveness of our newly invented sinus elevators (Fig. 1) by detaching goat maxillary sinus mucosa. In particular, the sinus mucosa detached in the mesial and distal directions or buccal and palatal directions was measured in length after the detaching operation for groups A, B, D and E. Meanwhile, the space volume created under the detached sinus mucosa was determined in groups $\mathrm{C}$ and $\mathrm{F}$.

Table 1 summarized the results for detaching goat maxillary sinus mucosa ex vivo using two designed shape-memory $\mathrm{Ni} / \mathrm{Ti}$ alloy wire containing tube elevators, which were different in the diameters of the shape-memory $\mathrm{Ni} / \mathrm{Ti}$ alloy wires used. One (namely elevator 012 ) had a shape-memory $\mathrm{Ni} / \mathrm{Ti}$ alloy wire with a diameter of 0.012 inch, while the other (namely elevator 014 ) had its shape-memory $\mathrm{Ni} / \mathrm{Ti}$ alloy wire with a diameter of 0.014 inch. Elevator 012 could detach the goat maxillary sinus mucosa in the mesial or distal direction for $12.1 \pm 4.3 \mathrm{~mm}$, while in the buccal or palatal direction for $12.5 \pm 6.7 \mathrm{~mm}$. The elevator 014 could detach the goat maxillary sinus mucosa for $23.0 \pm 4.9 \mathrm{~mm}$ in the mesial or distal direction, and for $19.0 \pm 8.1 \mathrm{~mm}$ in the buccal or palatal direction. An average space

Fig. 1. The shape-memory $\mathrm{Ni} / \mathrm{Ti}$ alloy wire containing tube elevators and their working mechanism: a) tube elevator showing the primary detaching knife (the part between the two red lines in the picture); b) elevator 012 ; c) elevator 014 ; d) the sketch of designed elevator showing the main components (1: primary detaching knife; 2: tube; 3: holder; 4: spring wire; 5: scale; 6: scales; 7: stopper; 8: shape-memory $\mathrm{Ni} /$ Ti alloy wire.); e) the sketch of primary detaching (10: implant hole or transcrestal access; 11: maxillary sinus mucosa.); and f) the sketch of secondary detaching (9: residual alveolar bone).

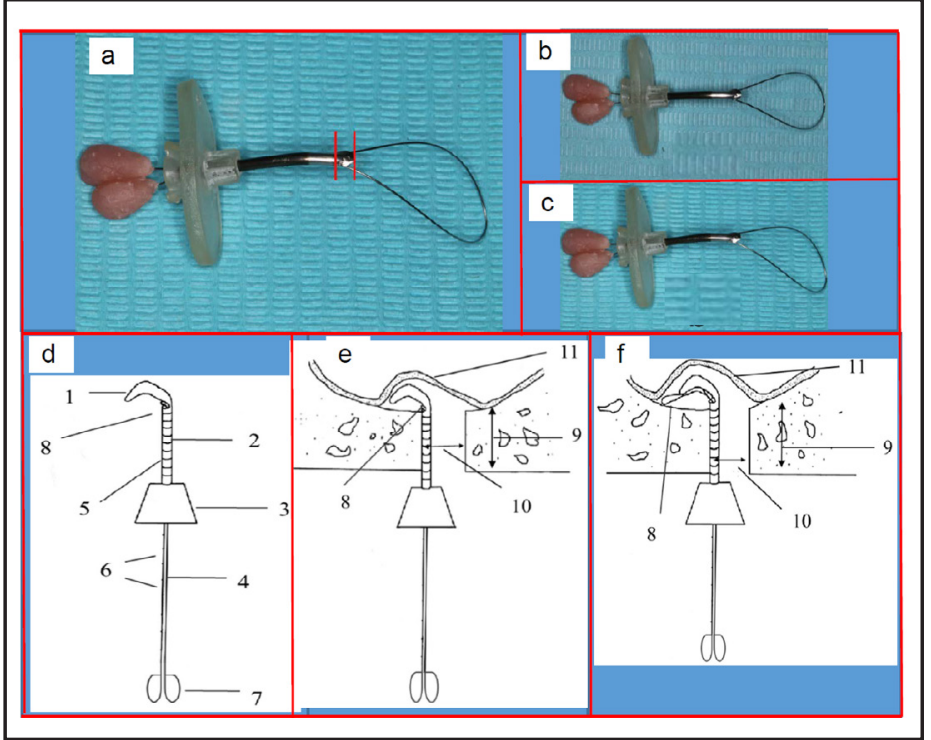




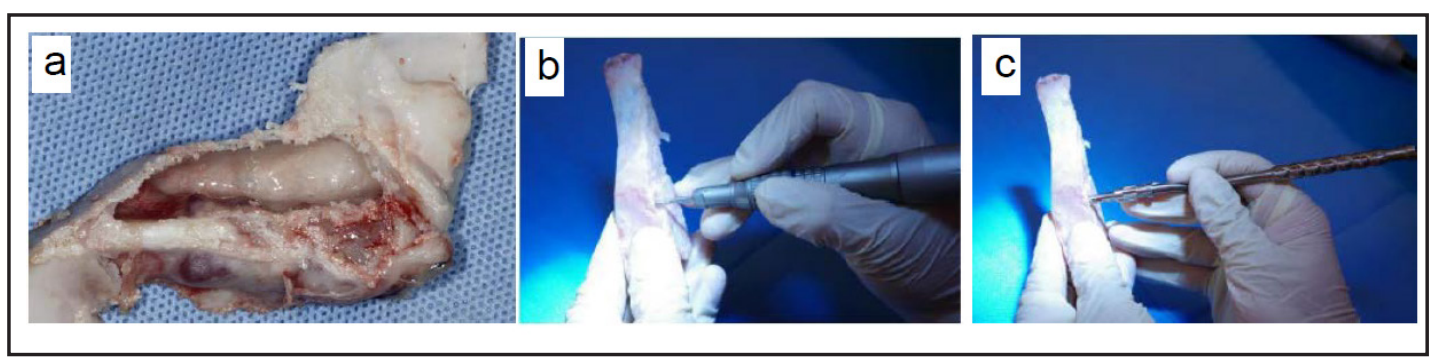

Fig. 2. The goat ex vivo maxillary sinus model: a) direct view of the sinus in goat ex vivo maxillary sinus model; b) $4 \mathrm{~mm}$ implant hole was prepared in the model; c) transcrestal access to maxillary sinus floor was prepared by removing the last 1-2 $\mathrm{mm}$ residual bone at the osteotomy site using osteotome technique.

Table 1.The ex vivo detaching efficacy using elevator 012 and elevator 014 . a. 2 perforation cases; b. 3 perforation cases; c. 1 perforation case; d. 1 perforation case

\begin{tabular}{lccc}
\hline Tool & $\begin{array}{c}\text { Length of sinus mucosa detached } \\
\text { in mesial/distal direction }(\mathrm{mm})\end{array}$ & $\begin{array}{c}\text { Length of sinus mucosa detached in } \\
\text { buccal/palatal direction }(\mathrm{mm})\end{array}$ & $\begin{array}{c}\text { Space volume created under } \\
\text { elevated sinus mucosa }(\mathrm{mm})\end{array}$ \\
\hline $\begin{array}{l}\text { Elevat } \\
\text { or } 012\end{array}$ & $12.1 \pm 4.3^{\mathrm{a}}$ & $12.5 \pm 6.7^{\mathrm{b}}$ & $1.7936 \pm 0.2079$ \\
$\begin{array}{l}\text { Elevat } \\
\text { or } 014\end{array}$ & $23.0 \pm 4.9 \mathrm{c}$ & $19.0 \pm 8.1^{\mathrm{d}}$ & $1.8764 \pm 0.2366$ \\
\hline
\end{tabular}

Fig. 3. The real time visualization of wire during the operation of detaching sinus mucosa in goat ex vivo models: a) detaching sinus mucosa in mesial/distal direction; b) detaching sinus mucosa in buc$\mathrm{cal} /$ palatal direction.
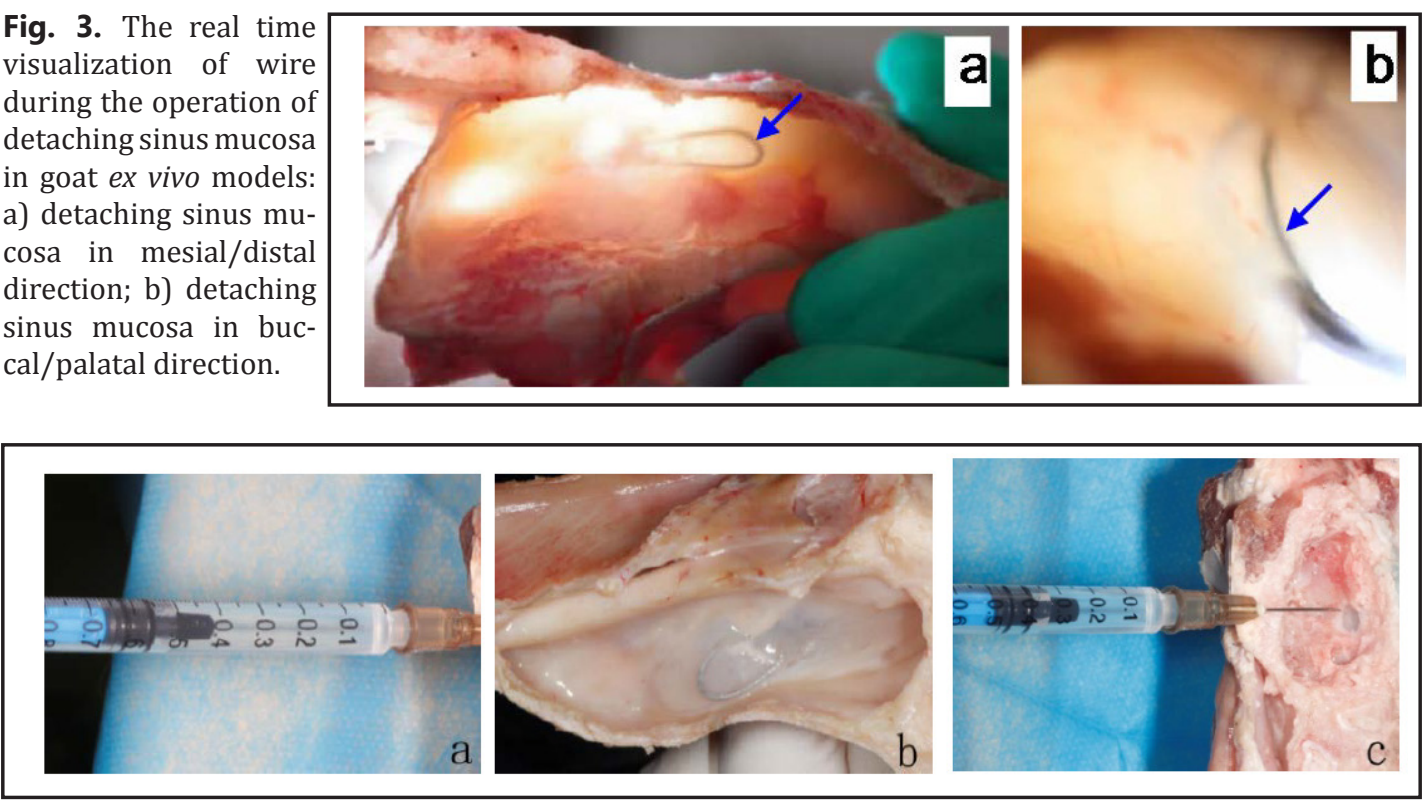

Fig. 4. The determination of space volume created under the elevated maxillary sinus mucosa: a) adding saline into the space before detaching maxillary sinus mucosa; b) detaching maxillary sinus mucosa ex vivo; c) adding saline into the space after detaching maxillary sinus mucosa.

volume of $1.7936 \pm 0.2079 \mathrm{ml}$ was created after detaching the goat maxillay sinus mucosa in both mesial/distal direction and buccal/palatal direction using elevator 012; while the average space volume created using elevator 014 was $1.8764 \pm 0.2366 \mathrm{ml}$. Fig. 3 showed the direct visualization of the sinus detaching process in real time. The shape-memory wire was directly seen beneath the lifted maxillary sinus mucosa.

When the two elevators were used to detach the sinus mucosa in the mesiodistal direction, two perforations (12.6\%) were observed for elevator 012 among the 16 ex vivo models (1 mesial direction and 1 distal direction for each goat ex vivo model) in group A, 


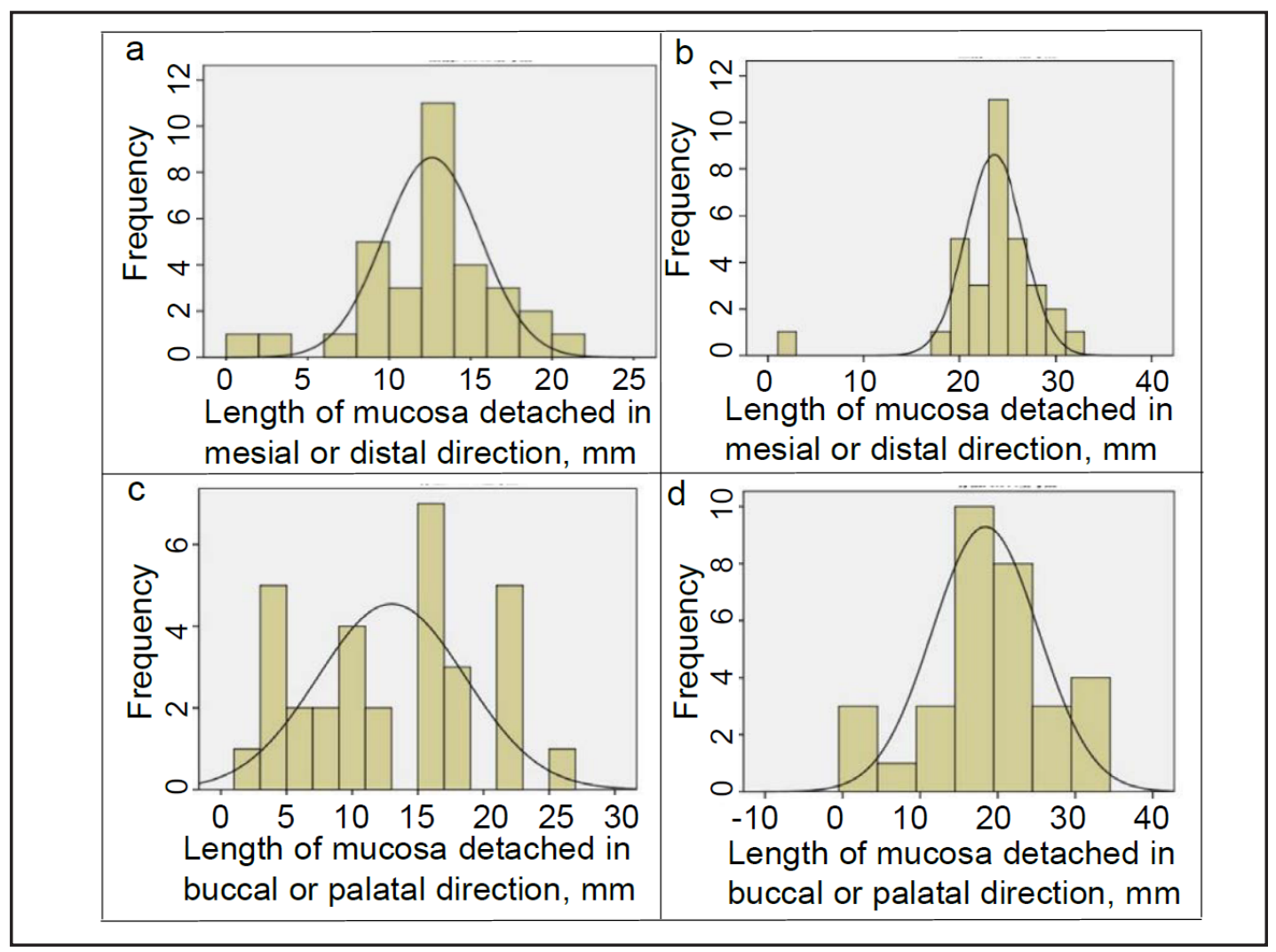

Fig. 5. The statistical distribution of lengths of maxillary sinus mucosa detached: a) using elevator 012 in mesial/distal direction; b) using elevator 014 in mesial/distal direction; c) using elevator 012 in buccal/ palatal direction; d) using elevator 014 in buccal/palatal direction.

while one perforation (6.25\%) was noticed for elevator 014 among the 16 ex vivo models in group D. The perforation occurred when the sinus mucosa was detached for about $1 \mathrm{~mm}$ using elevator 012; when elevator 014 was used the perforation happened when the sinus mucosa was detached for about $2 \mathrm{~mm}$. Moreover, in 7 cases, the front end of the wire reached the ends of the mesial or distal in group D using elevator 014 . We found statistical difference between groups A and D in the length of detached sinus mucosa $(p<0.05)$. All data in each group fitted the normal distribution (Fig. $5 a$ and $5 b$ ).

When the two elevators were used to detach the sinus mucosa in the buccal-palatal direction, three perforation cases (18.75\%) were observed for elevator 012 among the 16 ex vivo models in group B, while one perforation case $(6.25 \%)$ was noticed for elevator 014 among the 16 ex vivo models in group E. The perforation occurred when the sinus mucosa was detached for about 3-4 mm using elevator 012 , when elevator 014 was used the perforation happened when the sinus mucosa was detached for about $26 \mathrm{~mm}$. Thus, for the perforation in the group E, the sinus mucosa was almost completely detached when it was perforated. We found that there was statistical significant difference between groups B and $\mathrm{E}$ in the length of detached sinus mucosa $(p=0.001)$. All data in each group fitted the normal distribution (Fig. 5c and 5d).

For all the perforations occurred in groups A, B, and E, the sinus mucosa was perforated when it was detached for less than the average detached length of the corresponding particular group. We analyzed these failure cases and found that the failures were attributed to the fact that the residual 1-2 mm sinus floor bone was not completed pushed out from its position into the sinus cavity. This caused some difficulty to properly position the primary detaching knife during the initial detaching operation. For the perforation occurred in group F, the sinus mucosa was perforated when it was detached for more than the average

\section{KARGER}




\section{Cellular Physiology Cell Physiol Biochem 2016;40:944-952

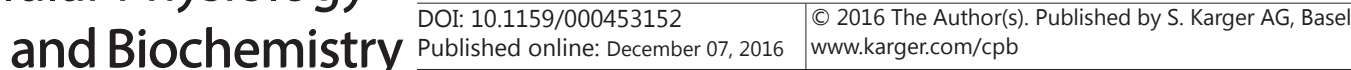

Li et al.: Ni/Ti Alloy Wire for Transcrestal Detaching Maxillary Sinus Mucosa

detached length of this group. This failure was attributed to the excessive detachment of the sinus mucosa.

When the two designed elevators were used to detach the sinus mucosa in both mesiodistal and buccal-palatal directions, we found no statistical difference between groups $\mathrm{C}$ and $\mathrm{F}$ in the space volume created under the elevated sinus mucosa $(p>0.05)$.

In order to achieve optimized clinical efficacy, continuous efforts have been made to improve the existing sinus lift tools or invent new sinus lift tools $[6,7,19,20]$. One golden standard for evaluating the effectiveness of a sinus lift tool is the length of maxillary sinus mucosa detached by the specific tool. In the transcrestal based maxillary sinus floor elevation, the transcrestal access to the maxillary sinus floor is limited by the size of the implant hole prepared in the maxilla. Using the traditional tools, it is difficult to detach the sinus mucosa to a large scale, thus limiting the volume generated and the height of the elevated sinus mucosa. In general, transcrestal maxillary sinus floor elevation using direct contacting tools is limited to those cases which need only to lift the sinus floor for a height of $3 \mathrm{~mm}$ or less due to safety issues. However, if better direct contacting sinus lift tools could be used to detach the maxillary sinus to much larger scales, this height limit could be lifted further. Thus more clinical cases could be treated using the transcrestal approach to elevate the maxillary sinus floor using direct contacting tools. Therefore, we invented these shapememory $\mathrm{Ni} / \mathrm{Ti}$ alloy wire containing tube elevators for detaching maxillary sinus mucosa via transcrestal approach. Our newly invented tube elevator consists of primary detaching tool and secondary detaching tool as shown in Fig. 1a. Primary detaching tool consists of tube and detaching knife at the front end, while the secondary detaching tool is the shapememory Ni/Ti alloy wire. The primary detaching tool would be used to detach the sinus mucosa right around the implant hole to open some gaps between the sinus bone and the sinus mucosa (Fig. 1e). The secondary tool would be used to further detach sinus mucosa far away from the implant hole after some gaps are created around the implant hole using the primary tool (Fig. 4f). This wire could detach the sinus mucosa via the movements of protruding, rotation and turn over, thus extending the detached sinus mucosa to a much larger scale.

In the clinical practice, a space volume of $0.67 \pm 0.17 \mathrm{ml}$ has been created using the inflatable balloon technique via the transcrestal approach to elevate the maxillary sinus floor [23]. Pommer et al. was able to produce a space volume of 0.9-3.1 ml under the lifted sinus mucosa in fresh human skulls using the gel-pressure technique via the transcrestal approach [21]. In this study, we were able to generate an average space volume of $1.7936 \mathrm{ml}$ using elevator 012 and $1.8764 \mathrm{ml}$ using elevator 014 . Due to the differences between human and goat, it would be impossible to directly compare our results with those reported in the literatures such as the two studies mentioned above. Our newly designed elevators might have some advantages in the application for detaching maxillary sinus mucosa. However, although the elevator 014 could detach the maxillary sinus mucosa to greater extents than the elevator 012 does, no statistical difference was noticed for the space volumes created under the sinus mucosa elevated by these two elevators (Table 1). This might be attributed to the great elasticity for maxillary sinus mucosa, the incapability of saline to infiltrate some space in the edges, and the morphology of the maxillary sinus floor. When elevator 014 was used to detach the sinus mucosa, it almost reached the end of the sinus. The morphology at these edge places might limit the detached mucosa to be fully expanded by the low density saline. Thus, the volume measured might be much less than the actual volume created after the sinus mucosa was detached using elevator 014.

\section{Conclusions}

The incidence rate of a conventional OSFE procedure can be up to 58\% (24), while our novel tube elevators could not only effectively detach the maxillary sinus mucosa on the goat ex vivo sinus models, but also significantly reduce the incidence of membrane perforation. 


\section{Cellular Physiology Cell Physiol Biochem 2016;40:944-952 \begin{tabular}{l|l|l} 
and Biochemistry $10.1159 / 000453152$ & (c) 2016 The Author(s). Published by S. Karger AG, Basel \\
Published online: December 07, 2016
\end{tabular} \\ Li et al.: Ni/Ti Alloy Wire for Transcrestal Detaching Maxillary Sinus Mucosa}

Moreover, the elevator containing shape-memory $\mathrm{Ni} / \mathrm{Ti}$ alloy wire with a diameter of 0.014 inch (namely elevator 014 ) has advantages over the other one (elevator 012) for the capability of detaching the sinus mucosa. The newly designed elevator 014 might be a promising tool for detaching maxillary sinus mucosa in the transcrestal maxillary sinus floor elevation.

\section{Acknowledgement}

This study was funded by the Clinical Support of PLA General Hospital (2014FCSXYY-1003) and the Capital's Special Health Development Research (2014-4-5022).

\section{Disclosure Statement}

The authors declare there is no conflict of interest.

\section{References}

1 Joos U, Kleinheinz J: Reconstruction of the severely resorbed (Class VI) jaws: Routine or exception? J Craniomaxillofac Surg 2000;28:1-4.

2 Wehrbein H, Diedrich P: Progressive pneumatisation of the basal maxillary sinus after extraction and space closure. Fortschr Kieferorthop 1992;53:77-83.

3 Summers RB: A new concept in maxillary implant surgery: the osteotome technique. Compendium (Newtown, Pa.) 1994;15:152, 154-156, 158 passim; quiz 162.

4 Tatum Jr H: Maxillary and sinus implant reconstructions. Dent Clin North Am 1986;30:207-229.

5 Al-Dajani M: Recent Trends in Sinus Lift Surgery and Their Clinical Implications. Clin Implant Dent Relat Res 2016;18:204-212.

6 Schwarz L, Unger E, Watzek G, Pommer B: Novel devices to prevent membrane perforation in transcrestal sinus floor augmentation surgery. Recent Pat Biomed Eng 2013;6:179-187.

$7 \quad$ Hwang JH, Jung B-Y, Lim C-S, Cha IH, Park W: Posterior Maxillary Segmental Osteotomy Concomitant With Sinus Lift Using a Piezoelectric Device. J Oral Maxillofac Surg 2011;69:2339-2344.

8 Tilotta F, Lazaroo B, Gaudy JF: Gradual and safe technique for sinus floor elevation using trephines and osteotomes with stops: a cadaveric anatomic study. Oral Surg Oral Med Oral Pathol Oral Radiol Endod 2008;106:210-216.

9 Li Y, Lv Y, Lu Y, Zeng P, Zeng X, Guo X, Han W: Design and finite element analysis of a novel sliding rod microscrew implantation device for mandibular prognathism. Int J Clin Exp Med 2015;8:10687-10695.

10 Li Y, Wang N, Lu Y, Zeng P, Zeng X, Guo X, Han W: Comparative static force analysis of molar distalization by a novel sliding rod microscrew implantation jig and direct traction. J Biomater Tissue Eng 2015;5:493-498.

11 Sun XJ, Xia LG, Chou LL, Zhong W, Zhang XL, Wang SY, Zhao J, Jiang XQ, Zhang ZY: Maxillary sinus floor elevation using a tissue engineered bone complex with BMP-2 gene modified bMSCs and a novel porous ceramic scaffold in rabbits. Arch Oral Biol 2010;55:195-202.

12 Trombelli L, Minenna P, Franceschetti G, Minenna L, Farina R: Transcrestal sinus floor elevation with a minimally invasive technique. J Periodontol 2010;81:158-166.

13 Sohn DS, Lee JS, An KM, Choi BJ: Piezoelectric internal sinus elevation (PISE) technique: A new method for internal sinus elevation. Implant Dent 2009;18:458-463.

14 Ucer C: Nasal suction technique for maxillary sinus floor elevation: a report of 24 consecutive patients. Int J Oral Maxillofac Implants 2009;24:1138-1143.

15 Krasny K, Krasny M, Kamiński A: Two-stage closed sinus lift: a new surgical technique for maxillary sinus floor augmentation. Cell Tissue Bank 2015;16:579-585.

16 Zitzmann NU, Schärer P: Sinus elevation procedures in the resorbed posterior maxilla: Comparison of the crestal and lateral approaches. Oral Surg Oral Med Oral Pathol Oral Radiol Endod 1998;85:8-17.

17 Emmerich D, Att W, Stappert C: Sinus Floor Elevation Using Osteotomes: A Systematic Review and MetaAnalysis. J Periodontol 2005;76:1237-1251. 


\section{Cellular Physiology Cell Physiol Biochem 2016;40:944-952 \begin{tabular}{l|l|l} 
DOI: 10.1159/000453152 & O 2016 The Author(s). Published by S. Karger AG, Basel \\
and Biochemistry & $\begin{array}{l}\text { Published online: December 07, } 2016 \\
\text { www.karger.com/cpb }\end{array}$
\end{tabular} \\ Li et al.: Ni/Ti Alloy Wire for Transcrestal Detaching Maxillary Sinus Mucosa}

18 Garbacea A, Lozada JL, Church CA, Al-Ardah AJ, Seiberling KA, Naylor WP, Chen JW: The incidence of maxillary sinus membrane perforation during endoscopically assessed crestal sinus floor elevation: A pilot study. J Oral Implantol 2012;38:345-359.

19 Li Y: Tube elevator containing shape-memory alloy wire for detaching maxillary sinus mucosa. International patent application 2015;Application number: PCT/CN2015/089842:

20 Petruzzi M, Ceccarelli R, Testori T, Grassi FR: Sinus floor augmentation with a hydropneumatic technique: a retrospective study in 40 patients. Int J Periodontics Restorative Dent 2012;32:205-210.

21 Pommer B, Watzek G: Gel-pressure technique for flapless transcrestal maxillary sinus floor elevation: a preliminary cadaveric study of a new surgical technique. Int J Oral Maxillofac Implants 2009;24:817-822.

22 Stelzle F, Rohde M: Elevation forces and resilience of the sinus membrane during sinus floor elevation: preliminary measurements using a balloon method on ex vivo pig heads. Int J Oral Maxillofac Implants 2014;29:550-557.

$23 \mathrm{Hu}$ X, Lin Y, Metzmacher AR, Zhang Y: Sinus membrane lift using a water balloon followed by bone grafting and implant placement: a 28-case report. Int J Prosthodont 2009;22:243-247.

24 Lopez MA, Bassi MA, Confalone L, Carinci F: Maxillary sinus floor elevation via crestal approach: The evolution of the hydraulic pressure technique. J Craniofac Surg 2014;25:e127-e132.

25 Kim JM, Sohn DS, Heo JU, Park JS, Jung HS, Moon JW, Lee JH, Park IS: Minimally invasive sinus augmentation using ultrasonic piezoelectric vibration and hydraulic pressure: A multicenter retrospective study. Implant Dent 2012;21:536-542.

26 Chen L, Cha J: An 8-year retrospective study: 1,100 patients receiving 1,557 implants using the minimally invasive hydraulic sinus condensing technique. J Periodontol 2005;76:482-491.

27 Mazor Z, Kfir E, Lorean A, Mijiritsky E, Horowitz RA: Flapless approach to maxillary sinus augmentation using minimally invasive antral membrane balloon elevation. Implant Dent 2011;20:434-438.

28 Ahn SH, Park EJ, Kim ES: Reamer-mediated transalveolar sinus floor elevation without osteotome and simultaneous implant placement in the maxillary molar area: Clinical outcomes of 391 implants in 380 patients. Clin Oral Implants Res 2012;23:866-872.

29 Calin C, Petre A, Drafta S: Osteotome-mediated sinus floor elevation: a systematic review and metaanalysis. Int J Oral Maxillofac Implants 2014;29:558-576. 